\title{
DERIVATIVES OF POLYNOMIALS WITH POSITIVE COEFFICIENTS
}

\author{
A. K. VARMA
}

ABSTRACT. Let $P_{n}(x)$ be an algebraic polynomial of degree $n$ with positive coefficients. We set

$$
I_{n}=\frac{\left\|P_{n}^{\prime}(x) \omega(x)\right\|_{L_{2}[0, \infty)}}{\left\|P_{n}(x) \omega(x)\right\|_{L_{2}[0, \infty)}}
$$

In this work upper bounds of $I_{n}$ are investigated. We restrict ourselves here with the case $\omega(x)=x^{\alpha / 2} e^{-x / 2}$. Results are shown to be best possible.

1. The problems dealt with in the present paper are connected with the classical inequalities of A. Markov [5], P. Erdos [1], G. G. Lorentz [3], [4], G. Szego [7] and P. Turan [8]. First, we describe them briefly.

Theorem A (A. A. Markov). If $f(x)$ is a polynomial of degree $n$, such that $|f(x)| \leqslant 1$ for $a \leqslant x \leqslant b$, then

$$
\max _{a<x<b}\left|f^{\prime}(x)\right| \leqslant \frac{2}{b-a} n^{2} .
$$

Further, this bound is sharp, as can be seen by the example

$$
f(x)=T_{n}(2(x-a) /(b-a)-1)
$$

where $T_{n}$ is Chebyshev's polynomial.

For special polynomials, one can sometimes improve the estimate (1.1).

Next, two theorems are of this kind.

TheORem B (P. ERDOS). Let $f(x)$ be a polynomial of degree $n$ satisfying the inequality $|f(x)| \leqslant 1$ for $-1 \leqslant x \leqslant 1$. Suppose $f(x)$ has only real roots and no root inside $[-1,+1]$; then for $-1 \leqslant x \leqslant 1$,

$$
\left|f^{\prime}(x)\right| \leqslant \frac{1}{2} e n \text {. }
$$

This is the best possible result.

Inequalities of the same type (as in Theorem B) hold for the wider class of polynomials with positive coefficients in $1+x, 1-x$; that is, for the polynomials

$$
f(x)=\sum_{k+l<n} a_{k l}(1+x)^{k}(1-x)^{l}, \quad a_{k l} \geqslant 0, \quad-1 \leqslant x<1 .
$$

These polynomials were introduced by G. G. Lorentz [3] and studied extensively by J. T. Scheick [6]. The Lorentz theorem can be stated as follows.

Received by the editors April 21, 1980.

1980 Mathematics Subject Classification. Primary 26A60; Secondary 30A40. 
Theorem C (G. G. Lorentz). There exists a constant $C>0$ such that for each polynomial $f(x)$ of the form (1.3),

$$
\frac{\left\|f^{\prime}\right\|}{\|f\|} \leqslant C n, \quad n=1,2, \ldots,
$$

for the uniform norm on $[-1,+1]$.

In $1964 \mathrm{G}$. Szego [7] studied the order of magnitude of $\left\|f^{\prime}\right\| /\|f\|$ for unrestricted polynomials $f$ of degree $\leqslant n$ for the norm

$$
\|f\|=\sup _{x>0}\left|f(x) e^{-x}\right|
$$

on $(0, \infty)$. More precisely, he proved the following.

Theorem D (G. Szego). Let $f(x)$ be a polynomial of fixed degree $n$ and not vanishing identically. Then

$$
\frac{\left\|f^{\prime}\right\|}{\|f\|}<C n, \quad n=2,3, \ldots,
$$

holds (for the weighted uniform norm as defined by $(1.5)$ on $(0, \infty)$ ).

In 1968 G. G. Lorentz [4] considered the problem of G. Szego for the special polynomials with positive coefficients in $x$,

$$
f(x)=\sum_{k=0}^{n} a_{k} x^{k}, \quad a_{k}>0,
$$

and the norm of a function $f$ on $(0, \infty)$ is given by

$$
\|f\|=\sup _{x>0}\left|f(x) e^{-\omega(x)}\right|
$$

where $\omega$ increases on $(0, \infty)$. Then the Lorentz theorem can be stated as follows.

Theorem E (G. G. Lorentz). Let $\omega(x)$ satisfy the inequalities $\omega(x)-\omega(0)<$ $A x \omega^{\prime}(x), x \geqslant 0$, and $\omega^{\prime}(y) \leqslant A \omega^{\prime}(x), y \leqslant x$, for some constant $A>0$. Then for some constant $C>0$, the inequality

$$
\frac{\left\|f^{\prime}\right\|}{\|f\|} \leqslant C \frac{\left\|p_{n}^{\prime}\right\|}{\left\|p_{n}\right\|}, \quad p_{n}(x)=x^{n}
$$

is valid.

Other related interesting results are due to A. Zygmund [10], Hille, Szego and Tamerkin [2] and P. Turan [8], where similar problems are considered either in $L_{2}$ norm or even in $L_{p}$ norm.

We may note that Lorentz raised the problem of determining the best constant in (1.6) and for (1.9).

Now we state the main theorem of this paper. Let $S_{n}[a, b]$ be the set of all polynomials whose degree is $n$ and whose roots are all real and no root inside $(a, b)$. It is easy to see that if $p_{n} \in S_{n}[0, \infty)$ then it can be expressed in the form

$$
P_{n}(x)=\sum_{k=0}^{n} a_{k} x^{k}, \quad a_{k} \geqslant 0 \text { for } k=0,1, \ldots, n .
$$


We now state

THEOREM 1. Let $P_{n}(x)$ be an algebraic polynomial of degree $n$ with nonnegative coefficients. Then for $\alpha \geqslant(\sqrt{5}-1) / 2$

$$
\int_{0}^{\infty}\left(P_{n}^{\prime}(x)\right)^{2} x^{\alpha} e^{-x} d x \leqslant \frac{n^{2}}{(2 n+\alpha)(2 n+\alpha-1)} \int_{0}^{\infty} P_{n}^{2}(x) x^{\alpha} e^{-x} d x,
$$

equality holding for $P_{n}(x)=x^{n}$. For $0 \leqslant \alpha \leqslant \frac{1}{2}$ we have

$$
\int_{0}^{\infty}\left(P_{n}^{\prime}(x)\right)^{2} x^{\alpha} e^{-x} d x \leqslant \frac{1}{(2+\alpha)(1+\alpha)} \int_{0}^{\infty} P_{n}^{2}(x) x^{\alpha} e^{-x} d x .
$$

Moreover (1.10) is also best possible in the sense that for $P_{n}(x)=x^{n}+\lambda x$ the expression on the left can be made arbitrarily close to the expression on the right-hand side by choosing $\lambda$ positive and sufficiently large.

COROLlaRY. Let $P_{n}(x)$ be an algebraic polynomial of degree $n$ with nonnegative coefficients satisfying (1.8) with $a_{n}>0$. Then for $\alpha \geqslant(\sqrt{5}-1) / 2$ we obtain

$$
\int_{0}^{\infty} P_{n}^{2}(x) x^{\alpha} e^{-x} d x \geqslant\left(a_{n}\right)^{2} \Gamma(2 n+\alpha+1)
$$

equality holding for $P_{n}(x)=x^{n}\left(a_{n}=1\right)$.

Proof of this Corollary is as follows. Repeated application of the inequality (1.9) gives at once

$$
\begin{aligned}
& \frac{\int_{0}^{\infty}\left(P_{n}^{k}(x)\right)^{2} x^{\alpha} e^{-x} d x}{\int_{0}^{\infty} P_{n}^{2}(x) x^{\alpha} e^{-x} d x} \\
& \quad \leqslant \frac{n^{2}(n-1)^{2} \cdots(n-k+1)^{2}}{(2 n+\alpha)(2 n+\alpha-1) \cdot \cdots(2 n+\alpha-2(k-1))(2 n+\alpha-1-2(k-1))} .
\end{aligned}
$$

Next, we note that

$$
\int_{0}^{\infty}\left(P_{n}^{(n)}(x)\right)^{2} x^{\alpha} e^{-x} d x=\left(a_{n}\right)^{2}(n !)^{2} \Gamma(\alpha+1)
$$

Now, we substitute $k=n$ in (1.12) and use (1.13) which leads to (1.11).

2. Proof of Theorem 1. Let $P_{n}(x)$ be any algebraic polynomial of degree $n$ with positive coefficients. Then we may write

$$
P_{n}(x)=a_{n} x^{n}+P_{n-1}(x), \quad P_{n-1}(x)=\sum_{k=0}^{n-1} a_{k} x^{k}, a_{k}>0
$$

Using (2.1) and

$$
\int_{0}^{\infty} x^{k} e^{-x} d x=\Gamma(k+1)
$$


we obtain

(2.3)

$$
\begin{aligned}
\int_{0}^{\infty}\left(P_{n}^{\prime}(x)\right)^{2} e^{-x} x^{\alpha} d x= & \int_{0}^{\infty}\left(P_{n-1}^{\prime}(x)\right)^{2} e^{-x} x^{\alpha} d x \\
& +a_{n}^{2} n^{2} \Gamma(2 n+\alpha-1)+2 n a_{n} \int_{0}^{\infty} P_{n-1}^{\prime}(x) x^{n+\alpha-1} e^{-x} d x,
\end{aligned}
$$

and

$$
\begin{aligned}
\int_{0}^{\infty}\left(P_{n}(x)\right)^{2} e^{-x} x^{\alpha} d x= & a_{n}^{2} \Gamma(2 n+\alpha+1) \\
& +\int_{0}^{\infty}\left(P_{n-1}(x)\right)^{2} e^{-x} x^{\alpha} d x+2 a_{n} \int_{0}^{\infty} P_{n-1}(x) e^{-x} x^{n+\alpha} d x .
\end{aligned}
$$

Next we set

$$
b_{n}=\frac{n^{2}}{(2 n+\alpha)(2 n+\alpha-1)}
$$

and

$$
\lambda_{n}=2 n \int_{0}^{\infty} P_{n-1}^{\prime}(x) x^{n+\alpha-1} e^{-x} d x-2 b_{n} \int_{0}^{\infty} P_{n-1}(x) x^{n+\alpha} e^{-x} d x .
$$

From (2.3)-(2.6) we may conclude that

$$
\begin{aligned}
\int_{0}^{\infty}\left(P_{n}^{\prime}(x)\right)^{2} e^{-x} x^{\alpha} d x-b_{n} \int_{0}^{\infty}\left(P_{n}(x)\right)^{2} e^{-x} x^{\alpha} d x \\
=\lambda_{n} a_{n}+\int_{0}^{\infty}\left(P_{n-1}^{\prime}(x)\right)^{2} e^{-x} x^{\alpha} d x-b_{n} \int_{0}^{\infty}\left(P_{n-1}(x)\right)^{2} e^{-x} x^{\alpha} d x
\end{aligned}
$$

First, we claim that for $\alpha \geqslant 0$

$$
\lambda_{n} \leqslant 0, \quad n=1,2, \ldots
$$

From (2.1), (2.2) and (2.6) we obtain

$$
\lambda_{n}=\frac{2 n}{(2 n+\alpha)(2 n+\alpha-1)} \sum_{k=0}^{n-1} a_{k} \mu_{k n} \Gamma(k+n+\alpha-1), \quad a_{k}>0
$$

where

$$
\begin{aligned}
\mu_{k n} & =(2 n+\alpha)(2 n+\alpha-1) k-n(k+n+\alpha)(k+n+\alpha-1) \\
& =(k-n)\{n(n-k)+(2 \alpha-1) n+\alpha(\alpha-1)\}, \quad 0<k<n-1 .
\end{aligned}
$$

Clearly for $\alpha \geqslant 0$ we have

$$
\mu_{k n} \leqslant-\alpha(2 n+\alpha-1) \leqslant 0, \quad k=0,1, \ldots, n-1 .
$$

Thus (2.8) follows easily from (2.9) and (2.10). We also note that

$$
b_{n} \geqslant b_{n-1}, \quad \alpha \geqslant \frac{\sqrt{5}-1}{2}, \quad n=2,3, \ldots,
$$

and

$$
b_{n}<b_{n-1}, \quad 0<\alpha<\frac{1}{2}, \quad n=2,3, \ldots
$$


Using these ideas we can complete the proof of Theorem 1. For $\alpha>$ $(\sqrt{5}-1) / 2$ we use (2.7), (2.8) and (2.11) and we obtain for $k=2,3, \ldots, n$,

$$
\begin{aligned}
\int_{0}^{\infty}\left(P_{k}^{\prime}(x)\right)^{2} e^{-x} x^{\alpha} d x-b_{k} \int_{0}^{\infty}\left(P_{k}(x)\right)^{2} e^{-x} x^{\alpha} d x \\
<\int_{0}^{\infty}\left(P_{k-1}^{\prime}(x)\right)^{2} e^{-x} x^{\alpha} d x-b_{k-1} \int_{0}^{\infty}\left(P_{k-1}(x)\right)^{2} e^{-x} x^{\alpha} d x
\end{aligned}
$$

Adding all these equations we obtain

$$
\begin{aligned}
\int_{0}^{\infty}\left(P_{n}^{\prime}(x)\right)^{2} e^{-x} x^{\alpha} d x-b_{n} \int_{0}^{\infty}\left(P_{n}(x)\right)^{2} e^{-x} x^{\alpha} d x \\
<\int_{0}^{\infty}\left(P_{1}^{\prime}(x)\right)^{2} e^{-x} x^{\alpha} d x-b_{1} \int_{0}^{\infty}\left(P_{1}(x)\right)^{2} e^{-x} x^{\alpha} d x
\end{aligned}
$$

But a simple computation shows that if $P_{1}(x)=a_{1} x+a_{0}, a_{1}>0, a_{0}>0$ then

$$
\int_{0}^{\infty}\left(P_{1}^{\prime}(x)\right)^{2} e^{-x} x^{\alpha} d x<b_{1} \int_{0}^{\infty}\left(P_{1}(x)\right)^{2} e^{-x} x^{\alpha} d x
$$

equality holds if $P_{1}(x)=a_{1} x, a_{1}>0$. From (2.14) and (2.15) we obtain (1.2) as desired for $\alpha \geqslant(\sqrt{5}-1) / 2$. For the proof of (1.3) for $0<\alpha<\frac{1}{2}$ we use (2.7), (2.8) and (2.12). From these results it follows that

$$
\begin{aligned}
\int_{0}^{\infty}\left(P_{k}^{\prime}(x)\right)^{2} & e^{-x} x^{\alpha} d x-b_{k} \int_{0}^{\infty}\left(P_{k}(x)\right)^{2} e^{-x} x^{\alpha} d x \\
\leqslant & \int_{0}^{\infty}\left(P_{k-1}^{\prime}(x)\right)^{2} e^{-x} x^{\alpha} d x-b_{k-1} \int_{0}^{\infty}\left(P_{k-1}(x)\right)^{2} e^{-x} x^{\alpha} d x \\
& +\left(b_{k-1}-b_{k}\right) \int_{0}^{\infty}\left(P_{k-1}(x)\right)^{2} e^{-x} x^{\alpha} d x
\end{aligned}
$$

Also from (2.4) it follows that

$$
\int_{0}^{\infty} P_{k-1}^{2}(x) x^{\alpha} e^{-x} d x<\int_{0}^{\infty}\left(P_{k}(x)\right)^{2} x^{\alpha} e^{-x} d x
$$

From (2.16) and (2.17) we obtain for $k=2,3, \ldots, n-1, n$,

$$
\begin{aligned}
\int_{0}^{\infty}\left(P_{k}^{\prime}(x)\right)^{2} e^{-x} x^{\alpha} d x-b_{k} \int_{0}^{\infty}\left(P_{k}(x)\right)^{2} e^{-x} x^{\alpha} d x \\
<\int_{0}^{\infty}\left(P_{k-1}^{\prime}(x)\right)^{2} e^{-x} x^{\alpha} d x-b_{k-1} \int_{0}^{\infty}\left(P_{k-1}(x)\right)^{2} e^{-x} x^{\alpha} d x \\
+\left(b_{k-1}-b_{k}\right) \int_{0}^{\infty}\left(P_{n}(x)\right)^{2} e^{-x} x^{\alpha} d x
\end{aligned}
$$

We add all these equations for $k=2,3, \ldots, n-1, n$ and we obtain

$$
\begin{aligned}
\int_{0}^{\infty}\left(P_{n}^{\prime}(x)\right)^{2} e^{-x} x^{\alpha} d x-b_{n} \int_{0}^{\infty}\left(P_{n}(x)\right)^{2} e^{-x} x^{\alpha} d x \\
<\int_{0}^{\infty}\left(P_{1}^{\prime}(x)\right)^{2} e^{-x} x^{\alpha} d x-b_{1} \int_{0}^{\infty}\left(P_{1}(x)\right)^{2} e^{-x} x^{\alpha} d x \\
\quad+\left(b_{1}-b_{n}\right) \int_{0}^{\infty}\left(P_{n}(x)\right)^{2} e^{-x} x^{\alpha} d x \\
<\left(b_{1}-b_{n}\right) \int_{0}^{\infty}\left(P_{n}(x)\right)^{2} e^{-x} x^{\alpha} d x
\end{aligned}
$$


But clearly (2.18) is equivalent to

$$
\int_{0}^{\infty}\left(P_{n}^{\prime}(x)\right)^{2} e^{-x} x^{\alpha} d x \leqslant b_{1} \int_{0}^{\infty}\left(P_{n}(x)\right)^{2} e^{-x} x^{\alpha} d x .
$$

From (2.19) and (2.5) follows (1.3).

In order to show that (1.3) is best possible we consider $f_{0}(x)=x^{n}+\lambda x, \lambda>0$, $0 \leqslant \alpha \leqslant \frac{1}{2}$. A simple computation shows that

$$
\frac{\int_{0}^{\infty}\left(f_{0}^{\prime}(x)\right)^{2} e^{-x} x^{\alpha} d x}{\int_{0}^{\infty}\left(f_{0}(x)\right)^{2} e^{-x} x^{\alpha} d x}=\frac{1}{(\alpha+2)(\alpha+1)}-C_{n, \lambda},
$$

where

$$
C_{n, \lambda}=\frac{2 \lambda \Gamma(n+\alpha)\left[\alpha(\alpha+1)+n^{2}-n\left(\alpha^{2}+\alpha-1\right)\right]+d_{n}}{(\alpha+2)(\alpha+1)\left[\lambda^{2} \Gamma(\alpha+3)+2 \lambda \Gamma(n+\alpha+2)+\Gamma(2 n+\alpha+1)\right]}
$$

and

$$
d_{n}=\Gamma(2 n+\alpha-1)\left[n^{2}\left(2-3 \alpha-\alpha^{2}\right)+2 n(2 \alpha-1)+\alpha(\alpha-1)\right] .
$$

Clearly $C_{n, \lambda} \rightarrow 0$ as $\lambda \rightarrow \infty$ from which our assertion follows.

\section{REFERENCES}

1. P. Erdos, Extremal properties of derivatives of polynomials, Ann. of Math. (2) 41 (1940), 310-313.

2. E. Hille, G. Szego and J. D. Tamerkin, On some generalizations of a theorem of A. A. Markhoff, Duke Math. J. 3 (1937), 729-739.

3. G. G. Lorentz, The degree of approximation by polynomials with positive coefficients, Math. Ann. 151 (1963), 239-251.

4. __ Derivatives of polynomials with positive coefficients, J. Approx. Theory 1 (1968), 1-4.

5. A. A. Markov, On a problem of D. I. Mendeleev, Izv. Akad. Nauk 62 (1889), 1-24.

6. J. T. Scheick, Inequalities for derivatives of polynomials of special type, J. Approx. Theory 6 (1972), 354-358.

7. G. Szego, On some problems of approximations, Magyar Tud. Akad. Mat. Kutato Int. Dozl. 2 (1964), 3-9.

8. P. Turan, Remarks on a theorem of Erhard Schmidt, Mathematica 2 (25) (1960), 373-378.

9. A. K. Varma, Some inequalities of algebraic polynomials having real zeros, Proc. Amer. Math. Soc. 75 (1979), 243-250.

10. A. Zygmund, A remark on the conjugate series, Proc. London Math. Soc. 36 (1932), 392-400.

Department of Mathematics, University of Florida, Gainesville, Florida 32611 